\title{
3D multi-scale study on metal/polymer nano-composites
}

\author{
Manon Goubet ${ }^{1}$, Constantin Matei ${ }^{1}$, Zineb Saghi $^{2}$, Bernard Viala $^{1}$ and Jean-Herve Tortai ${ }^{3}$
}

${ }^{1}$ CEA-Leti, Université Grenoble Alpes, F-38000 Grenoble, France, Grenoble, Rhone-Alpes, France, ${ }^{2}$ CEALeti, Université Grenoble Alpes, F-38000 Grenoble, France, Rhone-Alpes, France, ${ }^{3}$ CNRS/LTM, Grenoble, Rhone-Alpes, France

Introduction: Metal-polymer nanocomposites are a special class of materials that are capable to achieve usually incompatible combinations of physical constants like a giant-permittivity (e) or a high-permeability at RF $(\mu)$ and no conductivity (s). The objective of this work is to study the size, shape, separation and integrity of the nanoparticles, and the dispersion quality and volume fraction contained in the polymer. These quantities play a key role on the electrical or magnetic properties of the nanocomposite films. Two-dimension (2D) information is obtained by scanning transmission electron microscopy (STEM) with nanoparticle suspensions directly deposited on carbon grids by spin coating. Three-dimensional (3D) information is achieved by focused ion beam (FIB) - scanning electron microscopy (SEM) tomography on cross-sectional samples of the nanocomposite film deposited by spincoating on a silicon wafer. $2 \mathrm{D}$ morphological analysis of the nanoparticles during formulation: Two types of TEM samples were taken during the nanocomposite formulation: after dispersion and after surface functionalization. The liquid samples were deposited on TEM grids by spin coating and dried on a hot plate. The observations were realized with a FEI Tecnai Osiris S/TEM operating at $200 \mathrm{kV}$. The 2D distribution of particle size was performed with IPSDK software [1]. The Highangle annular dark field STEM (HAADF-STEM) images were segmented and the nanoparticles were labelled to measure Feret diameter and circularity (see fig 3). Overlapping nanoparticles were considered as agglomerates during the segmentation step thereby the circularity was used to discriminate single nanoparticles. Nanoparticles' mean Feret diameter allows to confirm the supplier's data, here the mean diameter (28nm) for 28 nanoparticles labelled, which was conform to the diameters given by the supplier (30 to 50nm). 3D morphological analysis of the film using FIB-SEM tomography: The acquisition on the nanocomposite film was performed on a Zeiss Crossbeam 550, with a $4 \mathrm{~nm}$ isotropic voxel size and using an InLens secondary electron detector. The collected FIB-SEM volume was about $13 \mu \mathrm{m} \times 15 \mu \mathrm{m} \times 5 \mu \mathrm{m}$, for a two-day acquisition time. After acquisition, the stack was cropped and aligned in Fiji [2] with MultiStackReg plugin. Previous work on nanocomposite films was reported by Takacs \& al. in [3], where the segmentation process was made on Avizo software, based on gloabal thresholding and watershed. Nowadays, artificial intelligence allow new methods of image segmentation: machine learning and deep learning [4]. In this work, Ilastik [5] was used to segment the FIB-SEM volume. The pixel classification workflow (Pxl_C) is based on the random forest classifier algorithm, where the user chooses a pre-defined number of features and successive annotations are provided to train the classifier and assign features to pixels of the image. Once segmented, the nanoparticles were labelled by connected components in IPSDK. In parallel, the segmented nanoparticles were also classified and separated in the Object Classification workflow of Ilastik (Obj_C): two criteria for object detection and segmentation are applied to select objects with the highest probability of the nanoparticle class and preserve their shape. Pixel classification (Pxl_C) and object classification (Obj_C) workflows allow working on sub-volume before applying the segmentation on the total volume. Here, training was made on one sub-volume $(2 \mu \mathrm{mx} 2 \mu \mathrm{m} \times 200 \mathrm{~nm})$ for pixel classification and object classification before batch processing on the total volume $(13 \mu \mathrm{m} \times 15 \mu \mathrm{m} \times 5 \mu \mathrm{m})$. In order to select the more accurate description of the film properties, a comparative study have been made on Pxl_C and Obj_C segmentation efficiency. 3D rendering of the obtained volumes was made on Tomviz [6], see figure 2. IPSDK software was used to extract quantitative data from the sub-volume and the total volume of both segmentations. Obj_C seemed less sensitive to noise than Pxl_C. This can be explained by the fact that the Obj_C segmentation works on the probability of belonging of the object to the right category, whereas Pxl_C measured were based on the connected component labelling with no noise reduction step. More nanoparticles are detected in Pxl_C (19 098 in Pxl_C, 12315 in Obj_C), the mean Feret diameter is also inferior to Obj_C value (24,1nm in Pxl_C, 31,4 in Obj_C). The volume fraction 
remains nevertheless almost identical (1,18\% in Pxl_C, 1,07\% in Obj_C), meaning that the segmentation efficiency has a low impact on this parameter. This work is based on a machine learning approach for the segmentation of nanoparticles; another approach is possible with deep learning. The StarDist library allows the segmentation of convex objects in 2D and 3D images [7], [8]. Knowing that Co/C nanoparticles are spherical, this approach would give much more accurate results. Conclusion: A combination of 2D TEM and 3D FIB-SEM tomography techniques was used to characterize nanoparticles at different steps of the fabrication of a metal-polymer nanocomposite film. TEM samples of nanoparticle suspensions were prepared by direct spin coating on grids leading to a better characterization of 2D morphological parameters. FIB-SEM tomography using multiple film cross-sections benefited of improved post image processing resulting in more accurate 3D spatial information. Acknowledgment: This work was partly supported by PTA (Upstream Technological Platform), cooperated by CNRS RENATECH and CEA Grenoble, France

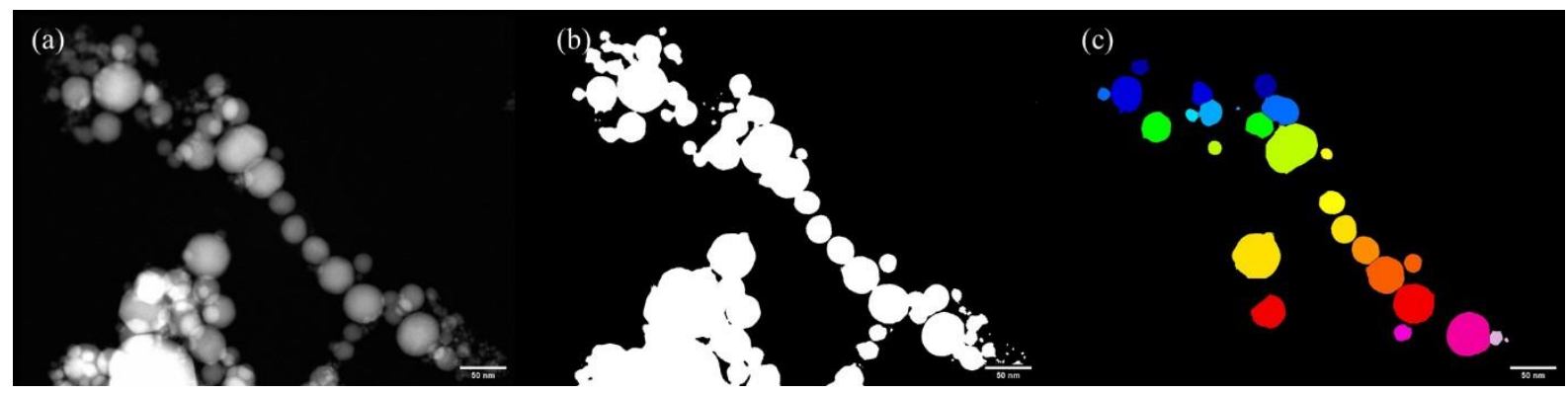

Figure 1. Figure 1: (a) HAADF-STEM image of Co@C//Pyr-PSa. (b) Segmented image. (c) Classified and labelled nanoparticles.

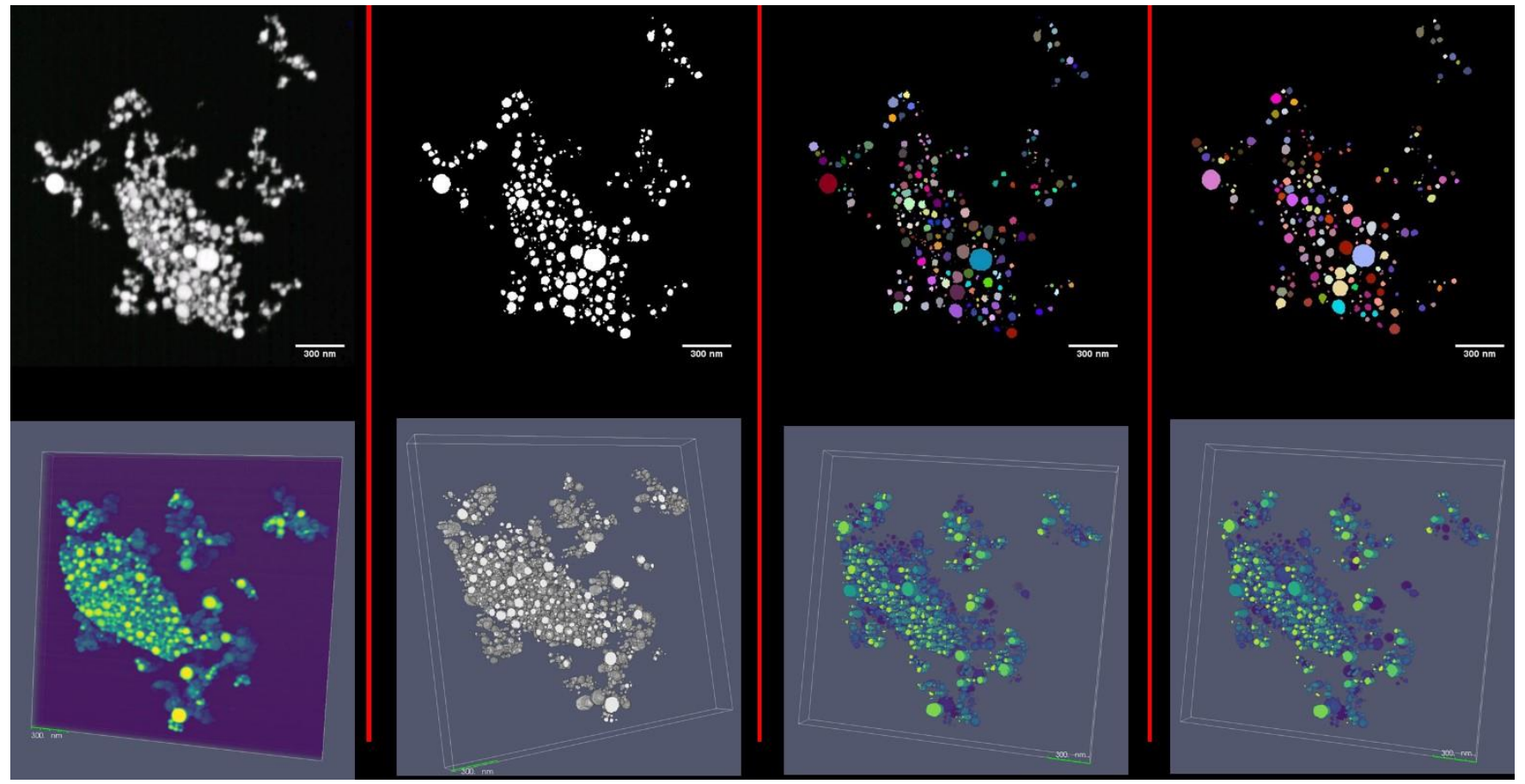

Figure 2. Figure 2 : Top (from left to right): a selected 2D FIB-SEM cross-section through the Co@C//PyrPSa volume; segmented image using random forest classifier (Ilastik); labelled image using connected component labelling (Pxl_C); labelled image using the object classification approach implemented in Ilastik. Bottom: 3D rendering of the selected sub-volume.

References 
[1] «IPSDK Smart Image Processing », Reactiv IP. https://www.reactivip.com/fr/accueil/ (consulté le févr. 18, 2021).

[2] J. Schindelin et al., « Fiji - an Open Source platform for biological image analysis », p. 15, 2013.

[3] H. Takacs et al., « Non-conductive ferromagnets based on core double-shell nanoparticles for radioelectric applications », SpringerPlus, vol. 5, nº 1, p. 496, déc. 2016, doi: 10.1186/s40064-016-2099-3.

[4] H. Seo et al., «Machine learning techniques for biomedical image segmentation: An overview of technical aspects and introduction to state-of-art applications », Medical Physics, vol. 47, n 5, p. e148-e167, 2020, doi: https://doi.org/10.1002/mp.13649.

[5] S. Berg et al., « ilastik: interactive machine learning for (bio)image analysis », Nat Methods, vol. 16, $\mathrm{n}^{\mathrm{o}}$ 12, p. 1226-1232, déc. 2019, doi: 10.1038/s41592-019-0582-9.

[6] B. D. A. Levin et al., «Tutorial on the Visualization of Volumetric Data Using tomviz », Microscopy Today, vol. 26, nº 1, p. 12-17, janv. 2018, doi: 10.1017/S1551929517001213.

[7] U. Schmidt, M. Weigert, C. Broaddus, et G. Myers, «Cell Detection with Star-convex Polygons », arXiv:1806.03535 [cs], vol. 11071, p. 265-273, 2018, doi: 10.1007/978-3-030-00934-2_30.

[8] M. Weigert, U. Schmidt, R. Haase, K. Sugawara, et G. Myers, « Star-convex Polyhedra for 3D Object Detection and Segmentation in Microscopy », in 2020 IEEE Winter Conference on Applications of Computer Vision (WACV), Snowmass Village, CO, USA, mars 2020, p. 3655-3662, doi: 10.1109/WACV45572.2020.9093435. 